\title{
Inclusión, Discapacidad y Profesores: Algunas Reflexiones para Repensar las Prácticas (Político- Educativas)
}

\section{Inclusion, Disability and Teachers: Some Reflections to Rethink Practices (Political-Educational)}

\author{
Rodolfo Cruz * \\ Universidad Popular Autónoma del Estado de Puebla, México
}

\begin{abstract}
El propósito específico de este texto es identificar ¿Qué papel juega la inclusión del estudiante con discapacidad en la constitución de un espacio inclusivo desde la perspectiva de los profesores? Esta pregunta surge del análisis de los resultados sobre los conflictos éticos que se presentan a los profesores en el proceso inclusivo escolar. En este sentido, se plantea partir desde una metodología de corte cualitativo con alcance descriptivo y desde un paradigma interpretativo, apoyándose con algunos elementos de la Teoría Fundamentada. Se utilizó un cuestionario con 13 preguntas abiertas realizadas a 61 profesores de educación obligatoria. El tipo de análisis que se dio a los resultados fue desde un sistema emergente y de discurso. Los resultados señalan que lo diferente, con su presencia, cuestiona y trastoca el orden establecido en las escuelas. Hace callar a los saberes y cuestiona las voluntades de verdad que se han establecido como únicas y siempre estáticas. Es así como la inclusión de la discapacidad, al interrogar el espacio, provocar el re-pensamiento de las acciones, conflictuar las prácticas de gobierno institucionales, interpelar a los agentes educativos y la comunidad en general, es una condición de posibilidad para transitar a esa escuela inclusiva que deseamos a nivel de las ideas.
\end{abstract}

Descriptores: Política de la educación; Estudiante; Discurso; Educación especial; Profesión docente.

The specific purpose of this text is to identify what role does the inclusion of the student with a disability play in the constitution of an inclusive space from the perspective of the teachers? This question arises from the analysis of the results on the ethical conflicts that are presented to the teachers in the inclusive school process. In this sense, it is proposed to start from a qualitative methodology with descriptive scope and from an interpretative paradigm, supporting with some elements of the Grounded Theory. A questionnaire was used with 13 free answer items to 61 teachers of compulsory education. The type of analysis that was given to the results was from an emerging system and discourse. The results indicate that what is different, with its presence, analyzes and disrupts the order established in schools. It silences the knowledge and questions the wills of truth that have been established as unique and always static. This is how the inclusion of disability, when questioning the space, provoking the rethinking of actions, conflicting institutional governance practices, questioning educational agents and the community in general, is a condition of possibility to travel to that inclusive school that we want at the level of ideas.

Keywords: Education policy; Student; Speech; Special education; Teaching Profession. 


\section{Introducción}

La violencia estructural, sistemática y simbólica (Bourdieu, 2009), las condiciones de precariedad, la pobreza, las situaciones de conflicto, entre muchos otros, son eventos que en su singularidad se presentan día a día como cuestión de cotidianidad (Escudero y Martínez, 2011).

En este marco frente a este proceso patologizante en lo personal e invisibilizante en lo social, se hace necesario replantear el papel que juega la educación en la actualidad. Mucho de lo que hoy se vive, como persona, (problemáticas sociales diversas), está íntimamente relacionado con las condiciones estructurales, con los mecanismos que intentan ordenar el espacio, pero también con los espacios mismos, a veces, escasos de resistencia. Aquí se hace imperante replantear los fines o finalidades, es decir, el lugar de la educación. Lo que interesa en este trabajo es la educación inclusiva y el espacio que debe ocupar como referente central para coadyuvar, desde su trinchera, a la constitución de relaciones menos violentas y asimétricas (Echeita, 2014; Giné, 2009; Ocampo, 2015; Slee, 2012).

El problema de investigación que se aborda en este trabajo tiene que ver con las posibilidades de la realización de una educación inclusiva que se pueden vislumbrar rescatando el aspecto relacional y ético más que el instrumental y pragmático. Cuando se habla de educación inclusiva, muchas veces se le coloca desde una visión práctica, donde lo importante tiene que ver con el establecimiento de diagnósticos y la asistencia a niños que presentan alguna problemática escolar, invisibilizando el papel que juega el posicionamiento del profesorado frente al estudiantado y los contenidos escolares. Por tanto, el supuesto que se defiende en este texto apunta a la necesidad de mirar las experiencias educativas inclusivas desde una matriz relacional e interactiva, donde la subjetividad, las emociones y los valores son los elementos centrales que pueden servir de condición de posibilidad para construir escuelas y espacios inclusivos.

Por tanto, en un primer momento se presenta un breve marco analítico-interpretativo sobre el significante educación inclusiva. Posteriormente la problematización del objeto de estudio, así como las preguntas y objetivos que guiaron al mismo. En otro momento se muestra el método desde el cual se ha emplazado este trabajo, así como el análisis de los resultados y su interpretación. Para finalizar, se cierra con algunas conclusiones, limitaciones y primeros hallazgos.

\section{Repensar la educación inclusiva: ¿Educación para todos, educación de calidad, educación democrática?}

La educación inclusiva hoy por hoy ocupa un espacio en los imaginarios educativos, donde se presenta como un tipo ideal que pueda llevar a la transformación de lo social y lo educativo. Dicho discurso parece posicionarse desde la construcción de un ideal de plenitud en donde la finalidad tiene que ver con la constitución de espacios simbólicos y terrenales en donde todos por igual puedan participar, estar y ser reconocidos en su individualidad, pero también como parte de una colectividad, en pocas palabras, una educación para todos de tinte democrático (Andrés y Sarto, 2009; Barrio, 2009; Castillo, 2015; Escudero y Martínez, 2011; Slee, 2012).

La cuestión en este punto es que, si bien la palabra democracia parece un significante de uso técnico, su utilización inmoderada en diversos espacios simbólicos la ha tornado casi 
una cuestión de sentido común. Poco se debate o cuestiona lo que se entenderá por "democrática", y los que lo hacen se conforman con hablar de igualdad, equidad y de un espacio donde no exista exclusión alguna.

En línea con lo anterior, lo democrático necesita ser repensado desde todo un corpus de conocimiento que se relaciona con la justicia, y aquí otro problema más, pues adentro de esta categoría habita una diversidad de significados, lo cual lo torna imposible de delimitar de forma simple. Si los significantes que se usan para referir a una educación inclusiva tienden a la polisemia y por ende a su escasa especificidad (Ainscow y Miles, 2009; Echeita, 2009), ¿cómo entender una educación que sea inclusiva desde un matiz democrático?

Hablar de educación para todos tampoco ayuda demasiado (Echeita, 2014), pues cuando se señala "Todos", en ese momento se pierde de vista la finalidad de dicha educación. En la actualidad, que todos reciban educación no implica que dicha educación sea la misma, en este sentido o bien tenemos una educación que responde a las necesidades de los estudiantes y que in situ está pensada para construir identidades en lo educativo que reflejen los principios de esa educación de carácter democrática, o bien sólo señalamos que hay una educación que puede ser considerada de calidad para unos, y otras que en diversa gradación pueden o no considerarse bajo ese adjetivo.

He aquí otra problemática ¿cuándo una educación puede ser considerada de calidad? Por un lado, se encuentra la educación que en cobertura llega a todos y que admite la presencia sin exclusión de los sujetos, no importa situación o condición, sin embargo, hasta ese momento no podemos señalar calidad alguna. Por otra parte, está la educación que pretende responder a las exigencias del entorno, un contexto cada vez más cambiante que invita a la formación de nuevas subjetividades que logren enfrentar los retos que, desde la exterioridad, representan una necesitad societal, no obstante, hasta este punto tampoco podríamos hablar de una educación de calidad. Pues cuando la persona que es educada no parece más que ser un ente reactivo a una fuerza del "afuera" que intenta sujetarlo y constituirlo en sujeto de desempeño, con una voluntad desde la eficiencia y respuesta, es decir, desde la competencia (Ainscow y Miles, 2009; Echeita, 2009), podría hablarse de un tipo de instrucción y capacitación, pero de educación, en un sentido amplio, difícilmente.

¿Dónde queda el sujeto en su capacidad de elegir, sentir, emocionarse, vivir? Parece entonces que falta un elemento de la fórmula para la calidad y tiene que ver con el propio sujeto en su necesidad, no solamente educativa, sino de vivir, ser y estar en determinado espacio. Sin embargo, dicho encuentro es el mayor reto, pues no aspira a la universalidad de las acciones sino todo lo contrario, intenta partir de la particularidad, de los deseos, de las voluntades, lo cual supera por mucho una educación que pretende enseñar al mismo tiempo, lo mismo a todos por igual. Otro paso hacia el concepto de calidad en la educación, podría ser retomado por lo que implica una educación inclusiva, pues ésta posee una alta relación semántica con la escuela democrática.

Hablar de educación inclusiva nos lleva muchas veces a la imprecisión lexical, es decir, hoy en día dicho significante se encuentra asociado a muchas temáticas, valores, pedagogías, metodologías e incluso filosofías, llevando a pensarlo hoy como un significante vacío.

Slee (2012, p. 102) presenta en este sentido algunas aproximaciones:

- La educación especial tradicional y sus antecedentes en medicina y psicología; 
- La teoría crítica y la nueva sociología de la educación y, en particular, la aplicación de este campo que hacen Barton y Tomlinson para facilitar una crítica pionera de las teorías y prácticas de la educación especial;

- Los estudios de la discapacidad y los estudios de la discapacidad en educación;

- El posestructuralismo, los estudios culturales y la teoría feminista;

- Los estudios poscoloniales, los estudios del desarrollo y la teoría crítica de la raza;

- La teoría política;

- La sociología política;

- La investigación sobre el currículum, la pedagogía y la evaluación (incluyendo la pedagogía crítica);

- La formación del profesorado;

- La geografía social;

- Los estudios sobre metodologías de investigación.

En este último punto, también se ubica una diversidad de trabajos que Slee (2012) organiza en tres ámbitos: En el primero, se encuentran los que vienen de la educación especial más tradicional a la que se puede considerar neoespecial, donde el interés tiene que ver con trabajar con necesidades educativas especiales para que, una vez comprendida la problemática, a partir del diagnóstico, se tengan claras las patologías, defectos, síndromes, etc.

En un segundo ámbito están los trabajos que realizan una fuerte crítica a toda la educación especial, estos más de carácter posmodernos, postestructuralistas y feministas, aquí todo el movimiento del modelo social de la discapacidad es uno de los mejores ejemplos.

En el tercer y último ámbito se encuentran los trabajos que se han dedicado a analizar los procesos de exclusión e inclusión desde la visión de las subjetividades existentes, por ejemplo, grupos de personas con discapacidad, indígenas, etc.

Por su parte, Giné (2009), retoma un trabajo de investigación realizado por Ainscow y César (2006) y Ainscow, Booth y Dyson (2006) donde, desde una visión internacional, encontraron seis maneras de entender la educación inclusiva.

- La educación inclusiva relacionada al tema de la discapacidad y las necesidades educativas especiales.

- Los problemas de conducta y la inclusión como respuesta y solución a los mismos.

- La inclusión como una forma de responder a grupos que se encuentran en riesgo de exclusión.

- La escuela para todos como forma de realización del proyecto inclusivo.

- La inclusión como sinónimo de escuela para todos.

- La inclusión como forma y principio que ayuda a entender nociones como educación y sociedad. 
Dentro de estos abordajes de la educación inclusiva, coexisten principios y fundamentos comunes, por lo que hace a esta tipología no excluyente entre sí. Lo que es visible es la polisemia de los significados que hoy tiene este significante y el problema en torno a qué estamos entendiendo desde las políticas educativas y los ordenamientos jurídicos, lo anterior sumado a las diversas traducciones que se realizan cuando estos postulados pretenden llevarse a lo concreto, es decir a las prácticas.

Bajo este espacio de amplia complejidad, en este trabajo, la educación inclusiva tendrá que ver con todo lo antes mencionado, reconociendo que cuando señalamos su carácter de democrático como una cualidad intrínseca a lo educativo, y considerando la participación social de todas las personas desde una perspectiva ética y de justicia, forzosamente se hace necesario acudir a analizar las relaciones que al interior de los procesos inclusivos se constituyen. Es decir, en qué otro espacio o ámbito se podría pensar y expresar su complejidad si no en los encuentros intersubjetivos (Sañudo, 2018), en donde sin duda, se juegan los valores de justicia y democracia día a día.

El problema hasta este punto tiene que ver con las siguientes cuestiones: ¿en qué espacios deben darse dichas relaciones? ¿Bajo qué perspectivas ontológicas y epistémicas se deben guiar? ¿Cómo pensar al otro y su relación con nosotros? ¿Basta con que se encuentren siendo educados en los mismos espacios escolares? ¿Es lo mismo hablar de educación especial que de inclusiva? En esta relación con los otros ¿Cómo entender la discapacidad y a la persona en esa situación? ¿Qué papel juega la política educativa y todo el movimiento inclusivo en la constitución de identidades sociales? Preguntas que difícilmente pueden ser contestadas en su totalidad, pero desde las cuales se pueden iniciar procesos analíticos y reflexivos.

\subsection{Educación inclusiva y discapacidad: Una mirada a este problema}

Lo que aquí se intenta problematizar es el discurso de la educación inclusiva, pero específicamente las relaciones que entre profesores y estudiantes son reconocidas como punto de partida para configurar los procesos de subjetivación.

Como ya se había comentado, hablar de educación inclusiva puede estar relacionado con varios aspectos. Por ejemplo, tiene que ver con determinados valores como la igualdad, equidad, etc. (Valenciano, 2009; Vélez, 2013). Por otro lado, se relaciona con cuestiones éticas como el reconocimiento de la dignidad del ser humano como elemento nodal que supera la condición, situación o cualquier otra característica particular o colectiva (López Melero, 2012). Tiene que ver además con una cuestión escolar e institucional, donde se pueden rescatar los aprendizajes, las formas de participación, interacción etc. También está relacionada con determinados agentes, por ejemplo, no sólo los alumnos, sino también, los profesores, las familias, los pares, los directivos etc. (Dainéz y Naranjo, 2015; Stainback y Stainback, 201 1; Vlachou, 2007).

La educación inclusiva también está relacionada con un ejercicio deconstructivo, es decir una visión desde una perspectiva individual y esencialista a una donde el centro parece estar en otro lado, en un afuera que constituye dichas subjetividades (Thomas y Loxley, 2007).

Cuando se revisa la literatura y las investigaciones sobre este tema, se encuentra que, bajo el significante de educación inclusiva, subyacen una diversidad de nominaciones, algunas íntimamente relacionadas, pero otras, representan imbricaciones con discursos que no precisamente apuntan a un mismo posicionamiento epistemológico y ontológico. Así, 
hablar de educación especial e inclusiva suele constituir giros, puentes, y encuentros frontales interesantes, que hacen cuestionar dónde inicia un discurso y termina el otro (Cruz, 2018; Echeita, 2014; Thomas y Loxley, 2007).

Por otra parte, se encuentra un discurso inclusivo que tiene su relación con lo político, con ese espacio de resistencia a la objetivación que las políticas educativas pretenden establecer al constituirse como espacios de subjetivación e identitarios (Infante, 2010; Slee, 2012). Identidades que más que adecuarse como calca al espacio de enunciación del discurso político se resisten a su objetivación, intentando desbordar el significante bajo el cual el proceso de nominación los ha sujetado. En este sentido, la educación inclusiva también se relaciona con la constitución de identidades y subjetividades. El concepto de subjetividades tiene que ver con la territorialización simbólica dinámica y constante, donde los sujetos pueden ocupar una multiplicidad de posiciones en el campo y resistir a la norma que intenta ubicarlo, clasificarlo y dictarle una determinada ontología.

En este trabajo lo que interesa tiene que ver con un grupo de los considerados "vulnerables" y en cuyo caso representan una parte poblacional desde la cual se ha planteado el cambio paradigmático de la educación inclusiva. Es el tema de la discapacidad, la persona o como en este trabajo se señala, el sujeto con discapacidad y su inclusión en espacios escolares regulares el que interesa problematizar.

\subsection{Normativización, discapacidad e inclusión: Un problema de estudio}

La norma (Foucault, 2014), como principio, parece acontecer del lado del prejuicio (Arent, 1997), momento anterior que no permite muchas veces construir un juicio en torno a un determinado objeto. La discapacidad, a lo largo de la historia, ha sido cuestionada por el paradigma de la norma, del prejuicio, y del sentido común, miradas que ajustan la condición deficitaria, la carencia, la falta y la imposibilidad con la persona, negando muchas veces el papel que la exterioridad juega para obstaculizar o posibilitar la participación social de los sujetos.

La persona, el sujeto, la discapacidad, se piensan desde el cuerpo, lugar donde se lee, a modo de texto, el Pathos. Corporalidades que en su situación de fragilidad y vulnerabilidad son considerados desde la minusvalía, una condición portada por el sujeto y por ende responsabilidad de él.

Barcalett (2016), al abordar la normalidad como un tipo de mirada, señala la voluntad de regulación que dicho espacio simbólico determina. Regulación que cae sobre los cuerpos $\mathrm{y}$, al hacerlo, les señala un espacio, un lugar y una identidad, nombrando un tipo de subjetividad frágil por naturaleza, un tipo de voluntad identitaria que construye lo que nombra, es decir, en el momento que se da el acto de nominación, también se constituye el objeto nominado (Butler, 2002).

Es desde este marco como muchas veces se ha pensado (y se sigue haciendo) la discapacidad, negando la estructuralidad que juega con imponer, a través de la norma, una condición determinada en el campo social, dejando como "culpa" o "responsabilidad" única al sujeto nominado bajo el rubro de "discapacitado". Sin embargo, frente a esta visión e imaginario, algunos movimientos y revoluciones de pensamiento se han dado para repensar y desmontar una serie de representaciones sobre este significante (discapacidad), intentando realizar un ejercicio deconstructivo, donde ya no se interrogue por la normativización del cuerpo, el sujeto con discapacidad, sino más bien se interroguen los 
espacios y los sistemas de razón que han premiado una forma de estar en el mundo (lo normal) sobre otras que pudieran denominarse "diferentes".

En este sentido, el modelo social y de derechos sobre la discapacidad (Brogna, 2009; Palacios, 2008), ha intentado realizar un ejercicio de descentramiento del sujeto de la discapacidad, colocando al significante fuera del cuerpo y posicionándolo en clave de discriminación. En este sentido se puede entender que la discapacidad no es algo que el sujeto posee, sino que se presenta cuando el espacio social ha limitado la participación (en equidad e igualdad) de dicha persona, colocándolos en espacios segregados o excluyéndolos de la interacción y socialización con aquellos cuyos cuerpos no son considerados excedentes.

La escuela no ha sido aséptica a estas ideas sobre la discapacidad, por ejemplo, en el movimiento de la escuela especial, en su interés por "educar", ha subyacido un ideal y razonamiento donde el fin es llegar a la cura del déficit y reparar el cuerpo que vive enfermo y necesita sanar (Echeita, 2014; Slee, 2012).

Por otro lado, en lo que respecta a los principios y el cambio paradigmático que implica la educación inclusiva, se ha intentado tejer otro tipo de urdimbre simbólico, donde la mirada exige focalización sobre las cuestiones estructurales y no sobre los cuerpos y sujetos que portan deficiencia (Segura, 2017). Es desde esta perspectiva desde la cual se mira y enuncia al sujeto con discapacidad en su proceso de inclusión escolar.

En este marco, esta investigación tuvo como pregunta central: ¿Cuál es el papel que juega el aspecto relacional en la constitución de identidades de estudiantes con discapacidad en su proceso de inclusión a la escuela regular? El propósito específico de este texto es identificar ¿Qué papel juega la inclusión del estudiante con discapacidad en la constitución de un espacio inclusivo desde la perspectiva de los profesores?

Este trabajo se justifica en varios sentidos, uno político, pues el tema de la educación inclusiva está aparejado a lo político en lo social, es decir, las nominaciones y los tipos de ordenamientos que gubernamentaliza ${ }^{1}$ la política educativa impacta directamente en las lógicas de razonamiento que se aplican en los espacios institucionalizados y se designan en las relaciones, estrategias y prácticas frente a la diversidad.

Una social, puesto que sin duda la inclusión de los grupos en situación de vulneración es un tema amplio que necesita ser retomado por la investigación educativa con miras a constituir espacios inclusivos donde la eticidad del ser y la justicia social sean un punto nodal que articule las relaciones entre las personas.

Una justificación también educativa, puesto que trabajos de este corte pueden coadyuvar a la reflexión de las prácticas y relaciones que los profesores establecen con sus estudiantes sea cual sea la situación o condición de éstas, asumiendo que por relación se entiende algo más que meras competencias técnicas e instrumentales que hoy se relacionan con la docencia.

\footnotetext{
${ }^{1}$ Las políticas son la nueva forma de gubernamentalidad, centradas en la población, pretenden objetivar y subjetivar la representación del deber ser del sujeto. Debemos entender que la política educativa entra en este esquema y por tanto es importante abordarla no sólo desde una visión superficial, sino rescatar el análisis en tres niveles. Llegar al plano ontológico y epistémico. Ver desde lo político a la política. Revisarla desde un juego de inclusión y exclusión.
} 


\section{Método}

Esta investigación se llevó a cabo en una institución de educación superior del Estado de Puebla, México. Tuvo un alcance descriptivo y fue realizada bajo una perspectiva cualitativa e interpretativa. La técnica fue el cuestionario cualitativo con 13 preguntas abiertas, el cual fue aplicado a 61 profesores de Educación Obligatoria del estado de Puebla (cuadro 1). Los datos fueron transcriptos fielmente para poder realizar, en un primer momento el análisis de contenidos de los mismos. Cabe hacer mención que previo a la aplicación de cuestionario se realizó un piloteo con 10 profesores. Al revisar los resultados de identificaron mejoras que podrían realizarse, además de disminuir el número de preguntas que en algunas resultaban reiterativas, teniendo, en un principio 18 preguntas, se sintetizaron en 13 .

La muestra de los participantes se realizó con base en lo siguiente:

- Profesores de educación básica Obligatoria de Puebla (preescolar, primaria, secundaria y bachillerato).

- Estar cursando la maestría en el primer año, puesto que en su condición de nuevo ingreso se podrían rescatar sus experiencias y conocimientos previos a la formación del posgrado.

- Tener alguna experiencia como docentes con algún estudiante con discapacidad.

- Estar interesados en participar en el estudio.

La aplicación se realizó en el periodo de enero a mayo del año 2017, con una previa autorización y consentimiento firmado. Lo mostrado a continuación tiene que ver con los resultados de la aplicación del cuestionario cualitativo. Cabe mencionar que todos los participantes eran estudiantes de la Maestría en Pedagogía con línea terminal de Educación Especial de la Universidad Popular Autónoma del Estado de Puebla (UPAEP).

Cuadro 1. Caracterización de los participantes

\begin{tabular}{lc}
\hline NIVEL EDUCATIVO & No DOCENTES $^{\circ}$ \\
\hline Educación Básica & 47 \\
Educación Media Superior & 14 \\
\hline Fuente: Elaboración propia. &
\end{tabular}

Se elaboró una codificación colocando una "D" en referencia a su condición de docente, posteriormente se asignó el número de cuestionario dependiendo el turno en que fue aplicado.

De los 61 participantes, 47 son de educación básica, sin embargo, no todos laboran o imparten docencia en los mismos niveles. 16 son profesoras de sexo femenino de preescolar (5 de escuelas privadas y 11 de públicas), 20 son profesores de nivel primario (11 de escuelas privadas y 9 de públicas), donde 6 de ellos son de género masculino y el resto femenino. Un total de 10 profesores son de secundaria, 7 de género femenino y 3 masculino. En el caso del profesorado de media superior, pertenecen a varias modalidades: 9 son profesoras de escuelas generales ( 5 mujeres y 4 varones) y 5 son profesoras de escuelas técnicas, todos de escuelas públicas.

Fueron 13 preguntas las que conformaron el cuestionario inicial, sin embargo, para efectos de este texto, y con base en la información que los datos arrojaron, sólo se analiza un solo 
cuestionamiento que intenta indagar sobre aquellos conflictos éticos que, en el proceso de inclusión de estudiantes con discapacidad, los profesores han enfrentado, sobre todo porque interesa ver el papel de la inclusión del estudiante en el espacio escolar (cuadro 1).

Para el análisis de la información, el sistema fue emergente, para esto se utilizaron algunos elementos que son propuestos por el método de Teoría Fundamentada (Strauss y Corbin, 2002). El proceso de recolección de datos se llevó a cabo cuidando los momentos y espacios para que se llevara a cabo una recogida de los mismos, reduciendo cualquier tipo de violencia simbólica y sesgos; de igual forma, éstos fueron transcriptos fielmente atendiendo los sentidos que los profesores participantes intentaron expresar².

En el análisis de la información se construyeron a partir de los datos categorías emergentes que sirvieron de base para realizar el análisis de discurso presentado. En un primer momento se identificaron unidades de análisis mediante un primer ejercicio de codificación. Dichas unidades fueron clasificadas por algunos códigos temáticos. Para dicho análisis discursivo se utilizaron algunas preguntas resultado de esa primera codificación y la identificación de las unidades de análisis. ¿Cuáles son los antagonismos que se construyen en el discurso? ¿Cómo son los procesos de inclusión y de exclusión en el discurso? ¿Cuál es la ideología que está presente en el discurso? ¿Cuáles son las categorías que sirven como argumento y defensa del discurso? ¿Cómo se manifiesta un pensamiento hegemónico en el discurso? ¿En qué momento el discurso está constituyendo lo social? ¿Qué tipo de discursos están presentes? ¿En qué parte se hace presente el elemento histórico y cómo?

\section{Resultados}

Este apartado se ha organizado a partir de 2 unidades significantes encontradas: una primera que apunta al conflicto ético de los profesores frente a la inclusión del estudiantado con discapacidad, una segunda titulada "la discapacidad como elemento conflictivo".

\subsection{Algunos conflictos éticos}

Como ya se ha apuntado en apartados anteriores de este escrito, hablar de inclusión o educación inclusiva parece llevarnos a una diversidad de cuestiones en donde los derechos, la legalidad, lo democrático, la participación y los valores están en el centro de los referentes de aquello que pretenda considerarse inclusivo (García, 2017). Es de reconocer que, aunque en su polisemia y muchas veces escasa especificidad, dichos discursos han sido incorporados por los profesores de diversas formas, pues traducidos en políticas, han buscado la interpelación de los principios discursivos y su identificación con determinados valores, los cuales colocaron al docente frente a un deber ser, desde el cual muchos miden y valoran su práctica.

${ }^{2}$ Cabe mencionar que este trabajo tuvo como antecedente un proyecto de investigación que buscó "Analizar las creencias que sobre la inclusión de personas con discapacidad tienen los profesores al atender a un estudiante en situación de discapacidad". Con base en el cuestionario aplicado y las respuestas de los profesores, se construyó un nuevo problema de investigación, el cual se mostró principalmente en torno a los conflictos éticos de la inclusión desde la percepción de los profesores. Lo que aquí se muestra es un nuevo apartado que busca problematizar la construcción del espacio inclusivo a partir de la presencia e inclusión de estudiantes con discapacidad al aula regular. De esta forma se analiza una pregunta del mismo cuestionario con un marco analítico e interpretativo diferente, pero utilizando elementos de la Teoría Fundamentada. 
En este sentido, los discursos de los profesores parecen oscilar, entre el deber ético y el peso que "injustamente" cae sobre el docente que no estaba preparado.

No creo que hay conflictos éticos, pues somos docentes preparados para resolver alguna problemática en determinada situación del contexto escolar. (D4)

No considero que haya conflictos éticos puesto que en la ética docente se incluye brindar educación a quien la necesite. (D40)

Asumir el compromiso de informarte para brindar el apoyo que se requiere y no por el simple hecho de que la persona tenga una discapacidad, proporcionar siempre una buena nota. (D54)

En un primer momento, el espacio inclusivo puede estar investido de un posicionamiento ético frente al otro, un compromiso que implica un tipo de subjetividad que está construida a partir de un deber ser profesional (Ethos), imperativo cubierto casi de un halo superior que, sin importar las condiciones y situaciones, es capaz de atravesar los riesgos y enfrentar las problemáticas.

No obstante, y como se verá a continuación, hay otros espacios discursivos que no implican precisamente ese deber ser y que más bien señalan la fragilidad de su subjetividad, la incompletud y la falta, aspecto que también se vuelve conflictivo para el profesorado al no poder posicionarse de forma frontal frente al otro, al considerarse incapaz de relacionarse. La presencia de ese resquebrajamiento que viene dado por la interrogación constante de lo "desconocido" lo "diferente", cuestiona la identidad del profesorado y lo coloca en el lugar de la incertidumbre, pero también de la injusticia, al haber sido arrojados sin formación alguna frente a algo que no comprenden y que estarían mejor con otras personas que "sí saben" tratarlos.

A no contar con el material, la capacitación, la forma de adecuación en la planeación y a la frustración por no saber si estás trabajando bien o mal. (D13)

El pensar si el alumno no podía estar mejor atendido en otro lado como con algún especialista.(D59)

Algunos profesores no quieren el trabajo extra que implica lograr una integración grupal teniendo a un alumno con discapacidad. (D41)

La falta de preparación, pues atendemos a alumnos en condiciones que no son las más adecuadas. (D55)

Como se puede observar, mucho de lo comentado en los discursos de los profesores apunta una situación conflictiva, donde la discapacidad y su inclusión parecen formularse como interrogación a los saberes y las prácticas y resulta en un extrañamiento a aquellos sujetos considerados diferentes. En este sentido, el conflicto, resultado de la introducción de más "complejidad", funciona como un elemento que activa lo político en el espacio escolar y que, como situación problematizante, re-elabora la discusión y el encuentro en torno al lugar y la subjetividad que está "capacitada", pero reconociendo también su compromiso en la tarea de educar a ese tipo de sujeto;

El docente busca medios, canaliza, brinda estrategias, adecua; pero no es el especialista, por lo que no tiene la facultad de hacer un diagnóstico sobre la situación del alumno y esto en ocasiones causa conflicto. Es que no tienen las bases para saber cómo tratar a estos alumnos y no saber cómo incluirlos con tantos niños diferentes. (D52)

Dicha situación está colmada de un tipo de encrucijada para el profesor, pues por un lado se asume carente, pero por el otro, reconoce lo humano como condición igual entre ese 
otro que es diferente a un propio "yo" constitutivo. Esto es visible en comentarios como los siguientes:

El dilema entre una intervención humana y una intervención profesional como docente. Hay cosas que no se pueden hacer porque la norma lo establece. (D20)

Éticos profesionales por cumplir con lo burocrático y qué puedo hacer para ayudar al alumno en su vida académica. Éticos personales: qué tanto estoy dispuesto a ofrecer de mí tiempo y esfuerzo para ayudar a este alumno. (D39)

$\mathrm{Al}$ parecer, la "intromisión" de la discapacidad implica también una reflexión personal, en donde se ponen a jugar varios elementos desde lo más humano y personal hasta lo más profesional y cuyo hilo conductor más bien tiene que ver con una determinada postura ética. Sin embargo, dicha postura no resuelve el conflicto (ético/moral) que se tiene, pues sustentada en una determinada norma moral, podrá ser habitada por una postura particular. En este caso, esto se refiere a un tipo de justicia escolar, donde se juega la presencia/ausencia del sujeto en torno a la respuesta educativa que "debería" en cuanto a su "condición" recibir.

Las preguntas y reflexiones de los profesores, son válidas, hasta cierto sentido, pues implican una mirada crítica al espacio "inclusivo" no constituido, donde se da la recepción del sujeto con discapacidad y el cual, a priori, carece de los elementos y recursos suficientes para tal acogida.

\section{Aceptarlo o no, por el miedo a no saber cómo actuar ante la discapacidad y si de verdad estoy haciendo lo correcto para ayudarlo. (D18) \\ Al no sentirse con la suficiente capacidad de lograr que el alumno se sienta incluido y sobre todo pueda trabajar con ellos. (D29)}

Sin embargo, también el espacio inclusivo se ha construido en dicha situación problemática desde otros planos y lugares de enunciación. Por ejemplo, desde un tipo de reconocimiento del otro, lo cual es señalado en un primer momento en los comentarios. Un tipo de mirada que equipara la posición social del sujeto en el campo escolar y lo sitúa de forma horizontal, como un par, como otro humano cuya diferencia más bien se diluye si pensamos en su potencialización (de la diferencia).

Como profesionistas no tendríamos por qué tener conflictos, al final, las personas comprenden, entienden y aceptan lo que conocen. (D50)

Que el trato sea igualitario con los alumnos, se debe cuidar que se les vea por igual, sin preferencias. (D31)

Éticos, ninguno porque se deben tratar por igual que otro alumno con los mismos valores y ética profesional. (D23)

Existe entonces un tipo de plano formal de la igualdad como punto de partida, un reconocimiento igualitario que, de entrada, coloca en una misma posición a la diversidad en el campo escolar y social. "D41 Comprender que también son personas normales". No obstante, lo conflictivo de esta postura es que, en realidad, desde una perspectiva inclusiva, no todos somos iguales, y más que hablar de igualdad habría que hablar de diferencia y desde la diferencia potenciar las oportunidades, pero desde una lógica equivalente (López Melero, 2011).

Lo anterior, también plantea otros conflictos en los profesores, pues si se considera que más que homogeneizar el espacio escolar, debo equiparar las oportunidades y hacerlas más bien equivalentes ¿cuál es el punto medio de dicho ejercicio? ¿En qué momento los procesos de diferenciación que se realizan permiten atender a la diversidad? y ¿hasta qué 
punto se retorna a otro tipo de violencia simbólica? (Bourdieu, 2009), en donde subyace no el reconocimiento del otro como mi igual, lo que es deseable en un primer momento, sino una constitución de la fragilidad representada en el sujeto con discapacidad.

A veces pensaba cuando tuve a mi alumna con discapacidad auditiva que le daba un trato más "cuidadoso" o especial a ella y a los demás de menor manera. (D51)

Quizás que minimizar al alumno y tratarlo como objeto. (D21)

La sobreprotección, la etiquetación o al no confiar en sus capacidades. (D42)

Apoyar a los mismos sólo por cumplir, aprobarlos al siguiente nivel académico siendo conscientes que no está preparado para hacerlo. (D26)

$A$ veces se descuida a los alumnos sin discapacidad por poner más atención al que cuenta con discapacidad. (D60)

Excluirlos cuando sabemos que ellos requieren más apoyo y atención que el resto. La falta de compromiso de darle acompañamiento óptimo. Al no saber cómo tratarlos, hablarles, sin dañar más su autoestima, su cuerpo o su mente. (D22)

Al parecer, se presenta un tipo de abundancia de precariedad que desborda la constitución identitaria del sujeto con discapacidad. Un tipo de equiparación escolar en donde la acción corresponde más bien a asumir un tipo de "pobreza pedagógica" (Escudero y Martínez, 2011) más que a un encuentro relacional entre subjetividades, más que un puente de apoyos relacionales.

Podemos ver que existe un tipo de interacción construida desde el espacio escolar, sin embargo, no así asegurar que dicho espacio pueda considerarse inclusivo, puesto que la respuesta educativa más bien está considerada como deficitaria en el sentido de las pocas expectativas de logro que se tienen y en la excesiva presencia de la constitución de subjetividades imposibilitadas, frágiles, carentes. Así la inclusión parece estar más bien travestida de integración escolar e incluso de un tipo de educación especial, pues el encuentro con el otro no se da, la relación de entrada es inexistente, el lazo es débil y se encuentra fracturado. Los comentarios apuntan no a la participación en comunidad sino en una separación dentro del espacio "inclusivo" en donde, por un lado, el docente atiende a los alumnos y por otro al que "posee" una discapacidad.

\subsection{La discapacidad y su inclusión como elemento conflictivo}

Es observable entonces como la educación inclusiva no es sinónimo de la presencia de la diferencia en los mismos espacios si éstos no se han equiparado para fomentar la participación de todos, si no se han construido desde la lógica de universalizar los diseños didácticos (Alba, 2016; Díez y Sánchez, 2015) y metodológicos para que todos puedan relacionarse. Tampoco podemos hablar de inclusión cuando las interacciones más que pensarse en presencias corporales en espacios similares, sean consideradas como encuentros relacionales de apoyo que, como finalidad última, sea la búsqueda de la autonomía y por ende la autodeterminación.

Es desde esta lógica en donde la preocupación de algunos docentes es loable, ¿cómo pensar la inclusión desde el mismo modelo pedagógico de la separación, ahora simbólica de las subjetividades? modelo que sigue ubicado desde la más tradicional lógica escolar de clasificación y por ende de posible estigmatización que lleva como efecto el continuo uso de las etiquetas escolares.

La señalización de personas y separación de seres humanos. No identificar que todos somos distintos, el maltrato. (D53) 
Etiquetarlos por su limitación. (D56)

El que llegamos a perder nuestra ética porque somos los primeros en etiquetar, segregar; en ocasiones de manera inconsciente o consideramos que tenemos mayor carga de trabajo. (D49)

Ser subjetivo y etiquetar, llegar a tratar de manera independiente y distinta al alumno, mostrar preferencias, desesperarse, etc. (D1)

En la realización de los diagnósticos y caer en la etiqueta de estos alumnos. (D57)

Regresando a la pregunta inicial ¿cómo se construye ese espacio inclusivo con la presencia del sujeto con discapacidad? Hasta este momento parece ser claro que el espacio es conflictivo, que la "inclusión" es precaria e incompleta, que la presencia no basta, pues la ausencia de un tipo de identidad que no se considere deteriorada está latente (Goffman, 2001). Lo político se hace presente en el encuentro con la discapacidad, la interrogación de los espacios, las prácticas, las acciones, las subjetividades son elementos altamente estables en las reflexiones éticas de los profesores.

No obstante, lo anterior, el conflicto es un buen inicio para poder emplazar el proceso constructivo de lo espacial-inclusivo como paradigma educativo. Sin embargo, es necesario trabajar en las preguntas y cuestiones que constituyen un apoyo para la inclusión y que no colocan la responsabilidad en el sujeto con discapacidad, en su deficiencia y su incapacidad y que más bien miran a la exterioridad que constituye día a día un tipo de identidad no frágil sino en proceso, en gerundio, un estar siendo humanos, una oportunidad para humanizarnos (López Melero, 2012). En este sentido es que el espacio inclusivo puede realizarse desde el reconocimiento de la propia no completud: “D14 Puede ser si ¿estoy haciendo lo correcto?”, el inicio por la duda no de otro sino de la relación con el otro.

La decisión y toma de postura personal sobre lo que debemos iniciar para que el proceso de inclusión se dé. El reto reflexivo que trae aparejado el conflicto de las presencias diversas, pero que al final representa un reto personal y profesional y una oportunidad de transformación del espacio, donde puede que no todo sea perfecto, pero en los avances y retrocesos, nos humanicemos todos.

No debería de haber conflictos éticos, si te toca, investigas cómo, pero lo haces, no hay maestros perfectos, tampoco alumnos idóneos y se debe trabajar con lo que nos den. (D45)

Considero que no son conflictos, sino retos que como docente adquieres con los alumnos con o sin discapacidad. (D5)

El principal, considero, es asumir el reto de prepararse y atender a las personas con discapacidad dentro del aula. (D9)

La mirada a la posibilidad, la actitud positiva hacia la diferencia, el intentar lo que a simple vista parece complicado y el reconocer que las fallas son parte del proceso, parecen ser elementos centrales para pensar los espacios inclusivos en las aulas e instituciones escolares. La humanización que se evidencia por las emociones que se pueden hacer presentes, "D38 Angustia". Pero que no anulan la acción, sino que en su aceptación se puede iniciar la transformación.

Este marco es la condición de posibilidad que debe terminar en el encuentro con el otro, en la relación de apoyo, relación que es el punto a valorar, para que, ni la responsabilidad sea del sujeto con discapacidad, ni del profesor en su totalidad, "D12 Se crea un sentimiento de culpabilidad cuando el alumno con discapacidad no ha tenido avances 
académicos"; sino más bien en un lugar intermedio, en la relación que se construye en el encuentro intersubjetivo (Orón, 2018).

\section{Conclusiones}

Como se había planteado desde un inicio, el propósito de este trabajo era contestar la siguiente pregunta: ¿Qué papel juega la inclusión del estudiante con discapacidad en la constitución de un espacio inclusivo desde la perspectiva de los profesores? En este sentido interesó ver ese proceso constructivo del espacio con la presencia del sujeto con discapacidad. A partir de los hallazgos se construyen tres categorías centrales para pensar estos procesos:

- Educación inclusiva ¿mismo juego, sólo nuevas reglas? En un primer momento, se pudo evidenciar cómo en lo educativo, aquello más tradicional, sigue presente en el denominado nuevo paradigma de educación inclusiva, si bien hay valores presentes, las prácticas y acciones pedagógicas lejos están de proceder casi de la forma más tradicional de pensar pedagógicamente. Hay separación, clasificación y segregación en la inclusión.

- ¿Educación de calidad o sólo educación para todos? En este marco habría que hacer las mismas preguntas de los docentes sobre si están o no los estudiantes recibiendo una educación de calidad, educación que los acerca cada día más hacia la autonomía.

- La discapacidad como principio político. Por otra parte, es de reconocer que la presencia de la diferencia en los espacios escolarizados ha funcionado desde el conflicto (lo político) como elemento interrogador que puede trastocar el orden establecido, poner en tensión los saberes más tradicionales y que en su iteración han sido sedimentados.

El espacio inclusivo entonces es posible construirlo desde el reconocimiento de la precariedad de todo el espacio, la humanización de las identidades y el afrontamiento de las responsabilidades que más que estar del lado de la ley, tienen un trasfondo ético en cuyo centro o punto nodal articulador se encuentra la dignidad de la persona. Es así como el papel de la discapacidad, del sujeto o persona con discapacidad es dinamizar y problematizar el espacio, desestabilizar para poder, a partir de la oportunidad de reacomodación, iniciar espacios y procesos inclusivos.

En línea con lo anterior, se tendría que reconocer que el punto de partida y llegada tiene que estar pensado en la relación, en la constitución de apoyos y puentes que permitan el encuentro de identidades. Cuando los profesores "reconocen" al otro y se miran desde un "nosotros", se puede hablar del inicio de una relación de apoyo. De la constitución de una capacidad (Nussbaum, 2012).

Para finalizar, lo retomado en este texto apunta a una serie de implicaciones, por un lado, el tema de la formación del profesorado, donde la visión educativa debe superar lo meramente instruccional, constituir experiencias formativas que tengan como centro la reflexión de las acciones, las prácticas y de los propios discursos que las guían y materializan. Por otro lado, está el tema desde las políticas educativas, al reconocer que no basta el establecimiento de ordenamientos jurídicos cuando éstos no han sido fruto de la participación de los encargados de llevarlas a cabo. 
En este sentido este trabajo puede continuarse intentando rescatar dichas prácticas, lo cual podría hacerse mediante un ejercicio de observación directa a los escenarios y espacios escolares, esto con la finalidad de observar e identificar cómo funcionan los mecanismos que se constituyen en las relaciones y que pueden posibilitar o no la empresa inclusiva. Es aquí donde se apunta una limitación de este trabajo, pues al sólo quedarse con los discursos de los profesores se pierde también la riqueza de las acciones.

\section{Referencias}

Ainscow, M. y Miles, S. (2009). Desarrollando sistemas de educación inclusiva. ¿Cómo podemos hacer progresar las políticas de educación? En C. Giné, D. Durán, J. Font y E. Miquel (Coords.), La educación inclusiva. De la exclusión a la plena participación de todo el alumnado. (pp. 161-170). Barcelona: Cuadernos de Educación.

Alba, C. (2016). Educación inclusiva y enseñanza para todos: El diseño universal para el aprendizaje. En C. Alba (Coord.) Diseño universal para el aprendizaje: Educación para todos y prácticas de enseñanza inclusivas (pp.15-25). Madrid: Morata.

Andrés, M. y Sarto, M. (2009). Escuela inclusiva: Valores, acogida y convivencia. En M. Sarto y M. Venegas (Coords.). Aspectos clave de la educación inclusiva. (pp. 85-117). Salamanca: INICO.

Arent, H. (1997). ¿Qué es la política? Barcelona: Paidós.

Barcalett, M. (2016). Una historia de la anormalidad. Finitud y ciencias en la obra de Michel Foucault. Ciudad de México: Gedisa.

Barrio, J. (2009). Hacia una educación inclusiva para todos. Revista Complutense de Educación, 20(1), 13-31.

Brogna, P. (2009). Las representaciones de la discapacidad: La vigencia del pasado en las estructuras sociales presentes. En P. Brogna (Comp.), Visiones y revisiones de la discapacidad, (pp. 157-187). Ciudad de México: Fondo de Cultura Económica.

Bordieu, P. (2009). El sentido práctico. Ciudad de México: Siglo XXI.

Butler, J. (2002). Cuerpos que importan. Sobre los limites materiales y discursivos del sexo. Ciudad de México: Paidós.

Castillo, C. (2015). La educación inclusiva y lineamientos prospectivos de la formación docente: Una visión de futuro. Revista Actualidades Investigativas en Educación, 15(5), 1-33. https://doi.org/10.15517/aie.v15i2.18534

Cruz, R. (2018). ¿Debemos ir de la educación especial a la educación inclusiva? Perspectivas y posibilidades de avance. Revista de Educación Alteridad, 13(2), 251-261.

Dainéz, D. y Naranjo, G. (2015). Los docentes ente las demandas de las políticas de educación inclusiva para la atención de niños con diferentes discapacidades: Casos México y Brasil. Revista Pro-Posiciones, 26(2), 187-204.

Díez, E. y Sánchez, S. (2015). Diseño universal para el aprendizaje como metodología docente para atender a la diversidad en la universidad. Aula Abierta, 43, 87-93. https://doi.org/10.1016/j.aula.2014.12.002

Echeita, G. (2009). Los procesos de inclusión educativa desde la declaración de Salamanca. Un balance entre doloroso y esperanzado. En C. Giné, D. Durán, J. Font y E. Miquel (Coords.), La educación inclusiva. De la exclusión a la plena participación de todo el alumnado (pp. 2547). Barcelona: Cuadernos de Educación. 
Echeita, G. (2014). Educación para la inclusión o educación sin exclusiones. Madrid: Narcea.

Escudero, J. y Martínez, B. (2011). Educación inclusiva y cambio escolar. Revista Iberoamericana de Educación, 55, 85-105.

Foucault, M. (2014). Los anormales. Ciudad de México: Fondo de Cultura Económica.

García, J. (2017). Evolución legislativa de la educación inclusiva en España. Revista Nacional e Internacional de Educación Inclusiva, 10(1), 21-264.

Giné, C. (2009). Aportaciones al concepto de inclusión. La posición de los organismos internacionales. En C. Giné, D. Durán, J. Font y E. Miquel (Coords.), La educación inclusiva. De la exclusión a la plena participación de todo el alumnado (pp. 13-24). Barcelona: Cuadernos de Educación.

Goffman, E. (2001). Estigma. La identidad deteriorada. Buenos Aires: Amorrortu.

Infante, M. (2010). Desafíos a la formación docente: Inclusión educativa. Revista Estudios Pedagógicos, 1, 287-297. https://doi.org/10.4067/S0718-07052010000100016

López Melero, M. (2011). Barreras que impiden la escuela inclusiva y algunas estrategias para construir una escuela sin exclusiones. Revista Innovación Educativa, 21, 37-54.

López Melero, M. (2012). La escuela inclusiva: Una oportunidad para humanizarnos. Revista Interuniversitaria de Formación del Profesorado, 74(26), 131-160.

Nussbaum, M. (2012). Crear capacidades: Propuesta para el desarrollo humano. Barcelona: Paidós.

Ocampo, A. (2015). Aproximaciones y descripciones generales sobre la formación del objeto de la educación inclusiva. En A. Ocampo (Coord.), Los rumbos de la educación inclusiva en Latinoamérica en los inicios del siglo XXI. Cartografías para modernizar el enfoque (pp. 24-90). Santiago de Chile: Celei.

Orón, V. (2018). Educación centrada en el crecimiento de la relación interpersonal. Revista Studia Poliana. Oviedo: Universidad de Navarra.

Palacios, E. (2008). El modelo social de discapacidad: Orígenes caracterización y plasmación en la convención internacional sobre los derechos de las personas con discapacidad. Ciudad de México: Ed. Cinca.

Sañudo, M. (2018). ¿Quién toma la palabra en la escuela, ¿Quién escucha? Preguntas pertinentes para la práctica reflexiva. Revista Latinoamericana de Educación Inclusiva, 12(1), 79-94. https://doi.org/10.4067/So718-73782018000100079

Segura, M. (2017). Elogio de la anormalidad. Revista Pasajes, 5, 40-45.

Slee, R. (2012). La escuela extraordinaria. Madrid: Morata.

Stainback, S. y Stainback, W. (2011). Aulas inclusivas. Un nuevo modo de enfocar y vivir el currículo. Madrid: Narcea.

Strauss, A. y Corbin, J. (2002). Bases de la investigación cualitativa. Técnicas y procedimientos para desarrollar la teoría fundamentada. Medellín: Universidad de Antioquia.

Thomas, G. y Loxley, A. (2007). Deconstrucción de la educación especial y construcción de la inclusiva. Madrid: La Muralla.

Valenciano, G. (2009). Construyendo un concepto de educación inclusiva: Una experiencia compartida. En M. Sarto y M. Venegas (Coords.), Aspectos clave de la educación inclusiva (pp. 14-24). Salamanca: INICO. 
Vélez, L. (2013). La educación inclusiva en docentes en formación: Su evaluación a partir de la teoría de facetas. Revista Folios, 37, 95-113. https://doi.org/10.17227/01234870.37folios95.113

Vlachou, A. (2007). Caminos hacia una educación inclusiva. Madrid: La Muralla.

\section{Breve CV del autor}

\section{Rodolfo Cruz}

Es Doctor en Investigación Educativa. Maestro en Educación. Especialista en Docencia por la UVM de Veracruz. Maestro en Necesidades Educativas Especiales. Especialista en Investigación Educativa. Actualmente realiza estudios de posdoctorado en la Unidad Interdisciplinaria de Ciencias de la Salud y Educación de la Universidad Nacional Autónoma de México (UNAM). Es Profesor-investigador en la Universidad Popular Autónoma del Estado de Puebla (UPAEP) Miembro del Sistema Nacional de Investigadores (SNI) nivel 1 del Consejo Nacional de Ciencia y Tecnología de México (CONACYT). Sus líneas de investigación son las políticas educativas sobre inclusión educativa y discapacidad. ORCID ID: https://orcid.org/oooo-0002-2561-1559. Email: rodolfo.cruz@upaep.mx 